\title{
GENERAL CONVERGENCE OF CONTINUED FRACTIONS
}

BY

LISA JACOBSEN

\begin{abstract}
We introduce a new concept of convergence of continued fractions-general convergence. Moreover, we compare it to the ordinary convergence concept and to strong convergence. Finally, we prove some properties of general convergence.
\end{abstract}

1. Introduction. A continued fraction

$$
K\left(a_{n} / b_{n}\right)=K \frac{a_{n}}{b_{n}}
$$

i.e.

$$
\begin{aligned}
K \frac{a_{n}}{b_{n}} & =\frac{a_{1}}{b_{1}}+\frac{a_{2}}{b_{2}}+\frac{a_{3}}{b_{3}}+\cdots \\
& =\frac{a_{1}}{b_{1}+\frac{a_{2}}{b_{2}+\frac{a_{3}}{b_{3}+.}}}, \quad a_{n} \in \mathbf{C} \backslash\{0\}, b_{n} \in \mathbf{C},
\end{aligned}
$$

is said to converge to a value $f \in \hat{\mathbf{C}}=\mathbf{C} \cup\{\infty\}$, if its sequence $\left\{f_{n}\right\}$ of approximants

$$
f_{n}=\frac{a_{1}}{b_{1}}+\frac{a_{2}}{b_{2}}+\cdots+\frac{a_{n}}{b_{n}}, \quad n \geqslant 1, f_{0}=0,
$$

converges to $f$. We write $\mathrm{f}=\lim f_{n}=K\left(a_{n} / b_{n}\right)$.

If $K\left(a_{n} / b_{n}\right)$ converges, then so do all of its tails

$$
f^{(n)}=\stackrel{\mathrm{K}}{\mathrm{K}=1}_{b_{n+m}}^{\infty} \frac{a_{n+m}}{b_{n+1}}+\frac{a_{n+2}}{b_{n+2}}+\frac{a_{n+3}}{b_{n+3}}+\cdots, \quad n \geqslant 0 .
$$

We see that $f^{(n)}$ is the value of the cutoff when we form the $n$th approximant $f_{n}$. In contrast to the tails of convergent series, for instance, $\left\{f^{(n)}\right\}$ does not usually converge to 0 . Hence, (1.2) is not necessarily the best choice of approximants of $K\left(a_{n} / b_{n}\right)$. In many cases it is better to use modified approximants

$$
S_{n}\left(w_{n}\right)=\frac{a_{1}}{b_{1}}+\frac{a_{2}}{b_{2}}+\cdots+\frac{a_{n}}{b_{n}+w_{n}}, \quad n \geqslant 1, S_{0}\left(w_{0}\right)=w_{0},
$$

where the modifying factors $w_{n}$ are chosen carefully. See, for instance, $[\mathbf{5}, \mathbf{8}]$.

Received by the editors January 7, 1985.

1980 Mathematics Subject Classification. Primary 40A15; Secondary 30B70.

Key words and phrases. Continued fractions, convergence, modified approximants. 
Considering modified approximants suggests a new definition of convergence of $K\left(a_{n} / b_{n}\right)$. This is illustrated in the following simple, but typical, examples.

EXAMPLE 1.1. The 3-periodic continued fraction

$$
K \frac{a_{n}}{1}=\frac{2}{1}+\frac{1}{1}-\frac{1}{1}+\frac{2}{1}+\frac{1}{1}-\frac{1}{1}+\frac{2}{1}+\cdots
$$

has the ordinary approximants

$$
f_{3 n-2}=\frac{2^{n}}{-1+\sum_{v=1}^{n} 2^{v}}=\frac{2^{n}}{2^{n+1}-3}, \quad f_{3 n-1}=\frac{2^{n}}{\sum_{v=1}^{n} 2^{v}}=\frac{2^{n-1}}{2^{n}-1}, \quad f_{3 n}=0
$$

for all $n \in \mathbf{N}$. (Here and in the rest of the paper $\mathbf{N}=\{1,2,3, \ldots\}$.) Clearly, $f_{3 n-2} \rightarrow 1 / 2, f_{3 n-1} \rightarrow 1 / 2$ and $f_{3 n} \rightarrow 0$ as $n \rightarrow \infty$. Hence, $K\left(a_{n} / 1\right)$ diverges (by Thiele oscillation [7, p. 87]). However, if we replace $\left\{f_{3 n}\right\}_{n=1}^{\infty}$ by modified approximants $S_{3 n}\left(w_{n}\right)$, where lim inf $\left|w_{n}\right|>0$, then, for sufficiently large $n$,

$$
S_{3 n}\left(w_{n}\right)=\frac{w_{n} 2^{n}}{1+w_{n} \sum_{v=1}^{n} 2^{v}} \rightarrow \frac{1}{2}
$$

Likewise,

$$
S_{3 n-2}\left(v_{n}\right)=\frac{2^{n}}{-1+\sum_{v=1}^{n} 2^{v}+v_{n}} \rightarrow \frac{1}{2} \quad \text { if } \lim \sup \left|v_{n}\right|<\infty
$$

and

$$
S_{3 n-1}\left(u_{n}\right)=\frac{2^{n}+u_{n} 2^{n}}{\sum_{v=1}^{n} 2^{v}+u_{n}\left(-1+\sum_{v=1}^{n} 2^{v}\right)} \rightarrow \frac{1}{2} \quad \text { if } \liminf \left|u_{n}+1\right|>0 .
$$

Thus $\left\{S_{n}\left(w_{n}\right)\right\}$ converges to $\frac{1}{2}$ for most choices of modifying factors $w_{n}$. Since $\left\{S_{n}(0)\right\}$ diverges, the divergence of the continued fraction appears to be an unfortunate result of the definition of convergence.

EXAMPLE 1.2. The 3-periodic regular $C$-fraction

$$
K \frac{a_{n} z}{1}=\frac{2 z}{1}+\frac{z}{1}-\frac{z}{1}+\frac{2 z}{1}+\frac{z}{1}-\frac{z}{1}+\frac{2 z}{1}+\cdots
$$

converges in a deleted neighborhood of $z=1$ to a holomorphic function [6, Theorem 3.1]. The singularity at $z=1$ is removable $\left(f(z):=\frac{1}{2}\right)$. The modified approximants $S_{n}(w)$, where $w=z+\frac{1}{2}$ for instance, converge, uniformly in compact subsets, in a neighborhood containing $z=1$ to the same holomorphic function.

In $\S 3$ we introduce a new definition of convergence (called general convergence) which includes the continued fractions in these examples but not the following one.

EXAMPLE 1.3. The 1-periodic continued fraction

$$
K \frac{a_{n}}{1}=\frac{-1}{1}+\frac{-1}{1}+\frac{-1}{1}+\cdots
$$

has the approximants $f_{n}=A_{n} / B_{n}$, where

$$
A_{3 n-2}=A_{3 n-1}=(-1)^{n}, \quad A_{3 n}=B_{3 n-1}=0, \quad B_{3 n}=B_{3 n+1}=(-1)^{n} .
$$


Hence, $K\left(a_{n} / 1\right)$ diverges. Moreover,

and

$$
\begin{gathered}
S_{3 n-2}(w)=\frac{(-1)^{n}}{(-1)^{n-1}(1+w)}=-\frac{1}{1+w}, \\
S_{3 n-1}(w)=\frac{(-1)^{n}(1+w)}{(-1)^{n-1} w}=-\frac{1+w}{w}
\end{gathered}
$$

$$
S_{3 n}(w)=\frac{(-1)^{n} w}{(-1)^{n}}=w \quad \text { for all } n .
$$

Hence, $\left\{S_{n}(w)\right\}$ diverges also, except if $w$ is chosen such that $\left\{S_{n}(w)\right\}$ is a constant sequence; that is, $w$ is a fixed point of $S_{1}(w)=-1 /(1+w)$. Even $\left\{S_{n}\left(w_{n}\right)\right\}$ diverges, except possibly if $\left\{w_{n}\right\}$ approaches a fixed point of $S_{1}(w)$.

Properties of general convergence are described in $\$ 4$. $\$ 2$ contains some historical remarks.

2. Historical remarks. The idea of considering different definitions of convergence of continued fractions is not a new one. Thron and Waadeland [8] pointed out that in 1918 Hamel [4] suggested a definition which took modified approximants into consideration. This definition was however quite vague.

In 1920 Hamburger [3] suggested

DEFINITION 2.1. A $J$-fraction $K\left(a_{n} /\left(b_{n}+z\right)\right)$ is said to converge completely in a set $D \subseteq \mathbf{C}$ if $\left\{S_{n}\left(t_{n}\right)\right\}$ converges uniformly in $D$ for every sequence $\left\{t_{n}\right\}, t_{n} \in \mathbf{R}$, to a function $f(z)$ independent of $\left\{t_{n}\right\}$.

Complete convergence was not intended to be used as a definition of convergence of a given continued fraction. It was a (very useful) tool in characterizing uniqueness of the solution of the Hamburger moment problem.

In 1982 Thron and Waadeland [8] made the following

DeFinition 2.2. We say that $K\left(a_{n} / b_{n}\right)$ converges strongly to a value $f \in \hat{\mathbf{C}}$ if

(i) there exist two distinct values $v, w \in \hat{\mathbf{C}}$ such that $f=\lim S_{n}(v)=\lim S_{n}(w)$, and

(ii) $L=\left\{z \in \hat{\mathbf{C}}\right.$; $z$ is a limit point of $\left.\left\{S_{n}^{-1}(\infty)\right\}_{n=1}^{\infty}\right\} \neq \hat{\mathbf{C}}$.

It is readily shown that a continued fraction can converge strongly to at most one limit. Thron and Waadeland [8] also proved

THEOREM 2.3. Let $K\left(a_{n} / b_{n}\right)$ be a continued fraction with $L \neq \hat{\mathbf{C}}$.

A. If $K\left(a_{n} / b_{n}\right)$ converges to $f$, then $K\left(a_{n} / b_{n}\right)$ converges strongly to $f$.

B. If $K\left(a_{n} / b_{n}\right)$ converges strongly to $f \neq \infty$, then $\lim S_{n}(w)=f$ for every $w$ such that $\operatorname{dist}(w, L)>0$.

By a straightforward argument one can see that the continued fraction in Example 1.1 converges strongly, whereas the one in Example 1.3 does not.

3. General convergence. Even though strong convergence is useful in many contexts, the sets $L$ may be difficult to characterize. Furthermore, we do not want to restrict ourselves to constant sequences of modifying factors. 
Definition 3.1. We say that $K\left(a_{n} / b_{n}\right)$ converges generally to a value $f \in \hat{\mathbf{C}}$ if there exist two sequences $\left\{v_{n}\right\},\left\{w_{n}\right\}$ of extended complex numbers such that

$$
\lim _{n \rightarrow \infty} S_{n}\left(v_{n}\right)=\lim _{n \rightarrow \infty} S_{n}\left(w_{n}\right)=f \text { and } \liminf _{n \rightarrow \infty} d\left(w_{n}, v_{n}\right)>0 .
$$

Here

$$
d(x, y)= \begin{cases}|x-y| 2 / \sqrt{1+|x|^{2}} \sqrt{1+|y|^{2}}, & x, y \in \mathbf{C}, \\ 2 / \sqrt{1+|x|^{2}}, & x \in \mathbf{C}, y=\infty, \\ 0, & x=y=\infty,\end{cases}
$$

is the chordal metric on the Riemann sphere [1].

By this definition we immediately get

THEOREM 3.2. If $K\left(a_{n} / b_{n}\right)$ converges to $f$ or converges strongly to $f$, then $K\left(a_{n} / b_{n}\right)$ converges generally to $f$.

Proof. If $K\left(a_{n} / b_{n}\right)$ converges to $f$, then $f=\lim S_{n}(0)=\lim S_{n}(\infty)$, and $v_{n}=0$, $w_{n}=\infty$ for all $n$ give general convergence. Similarly, if $\lim S_{n}(v)=\lim S_{n}(w)$ for $v \neq w$, then general convergence to $f$ follows.

In order to have full advantage of a definition of convergence of $K\left(a_{n} / b_{n}\right)$, we need uniqueness of the value.

THEOREM 3.3. If $K\left(a_{n} / b_{n}\right)$ converges generally to $f$ and to $g$, then $f=g$.

To prove this theorem as we shall use the following well-known result (see for instance [6, Theorem 2.1]):

$$
S_{n}\left(w_{n}\right)=\frac{A_{n}+A_{n-1} n_{n}}{B_{n}+B_{n-1} w_{n}}, \quad n=0,1,2, \ldots,
$$

where $A_{n}, B_{n} \in \mathrm{C}$ satisfy the linear recurrence relations

$$
Y_{n}=b_{n} Y_{n-1}+a_{n} Y_{n-2}, \quad n=1,2,3, \ldots,
$$

with initial conditions

$$
B_{-1}=A_{0}=0, \quad B_{0}=A_{-1}=1,
$$

and the determinant formula

$$
\Delta_{n}=A_{n} B_{n-1}-B_{n} A_{n-1}=-\prod_{m=1}^{n}\left(-a_{m}\right) \neq 0 .
$$

Hence, $S_{n}$ is a nonsingular linear fractional transformation which maps $\hat{\mathbf{C}}$ bijectively onto $\hat{\mathbf{C}}$. In the following we shall use the convention

$$
B_{n}+B_{n-1} x=B_{n} \quad \text { if } B_{n-1}=0, \quad \text { for all } x \in \hat{\mathbf{C}} \text {. }
$$

We shall furthermore use the notation

$$
h_{n}=-S_{n}^{-1}(\infty)=B_{n} / B_{n-1}, \quad n \in \mathbf{N} .
$$


The following lemma will be used in the proof of Theorem 3.3.

LEMMA 3.4.

$$
d\left(S_{n}\left(w_{n}\right), S_{n}\left(v_{n}\right)\right)= \begin{cases}\frac{2\left|\Delta_{n}\right|\left|w_{n}-v_{n}\right|}{C_{n}\left(w_{n}\right) C_{n}\left(v_{n}\right)} & \text { if } S_{n}\left(w_{n}\right) \neq \infty, S_{n}\left(v_{n}\right) \neq \infty, \\ \frac{2\left|B_{n}+B_{n-1} w_{n}\right|}{C_{n}\left(w_{n}\right)} & \text { if } S_{n}\left(w_{n}\right) \neq \infty, S_{n}\left(v_{n}\right)=\infty\end{cases}
$$

except if $v_{n}=w_{n}=\infty$, where

$$
C_{n}(x)=\sqrt{\left|A_{n}+A_{n-1} x\right|^{2}+\left|B_{n}+B_{n-1} x\right|^{2}} .
$$

Proof. Assume first that $S_{n}\left(w_{n}\right) \neq \infty$ and $S_{n}\left(v_{n}\right) \neq \infty$; that is, $B_{n}+B_{n-1} w_{n} \neq 0$, $B_{n}+B_{n-1} v_{n} \neq 0$. Then

$$
\left|S_{n}\left(w_{n}\right)-S_{n}\left(v_{n}\right)\right|=\frac{\left|\Delta_{n}\right|\left|w_{n}-v_{n}\right|}{\left|B_{n}+B_{n-1} w_{n}\right|\left|B_{n}+B_{n-1} v_{n}\right|},
$$

which gives (3.9) for this case by use of (3.2).

If $S_{n}\left(w_{n}\right) \neq \infty$ and $S_{n}\left(v_{n}\right)=\infty$, then $B_{n}+B_{n-1} w_{n} \neq 0$, and (3.9) follows by use of (3.2).

Proof of Theorem 3.3. Assume that, for some $d>0$ and $N \in \mathbf{N}$,

$$
\begin{array}{lll}
f=\lim S_{n}\left(v_{n}\right)=\lim S_{n}\left(w_{n}\right), & d\left(v_{n}, w_{n}\right) \geqslant d, & n \geqslant N, \\
g=\lim S_{n}\left(p_{n}\right)=\lim S_{n}\left(q_{n}\right), & d\left(p_{n}, q_{n}\right) \geqslant d, & n \geqslant N .
\end{array}
$$

If $p_{n} \neq v_{n}$ or $p_{n} \neq w_{n}$ or $q_{n} \neq v_{n}$ or $q_{n} \neq w_{n}$ for only a finite number of indices $n$, then clearly $f=g$. Assume that this is not so, and let $\left\{n_{k}\right\}_{k=1}^{\infty}$ be the subsequence of the natural numbers which contains all the indices for which

$$
p_{n_{k}} \neq v_{n_{k}}, w_{n_{k}} \quad \text { and } \quad q_{n_{k}} \neq v_{n_{k}}, w_{n_{k}}, \quad n_{k} \geqslant N
$$

It suffices to prove that, for $n=n_{k}$,

$$
P_{n}=d\left(S_{n}\left(w_{n}\right), S_{n}\left(q_{n}\right)\right) \cdot d\left(S_{n}\left(p_{n}\right), S_{n}\left(v_{n}\right)\right) \rightarrow 0 \quad \text { as } k \rightarrow \infty .
$$

Or, since

$$
Q_{n}=d\left(S_{n}\left(w_{n}\right), S_{n}\left(v_{n}\right)\right) \cdot d\left(S_{n}\left(p_{n}\right), S_{n}\left(q_{n}\right)\right) \rightarrow 0 \text { as } k \rightarrow \infty,
$$

it suffices to prove that $\left\{P_{n} / Q_{n}\right\}$ is bounded from some $n$ on. Since $p_{n}, q_{n}, v_{n}$ and $w_{n}$ are all distinct for $n=n_{k}$, it follows from Lemma 3.4 and (3.2) that

$$
\frac{P_{n}}{Q_{n}}=\frac{\left|w_{n}-q_{n}\right|}{\left|w_{n}-v_{n}\right|} \frac{\left|p_{n}-v_{n}\right|}{\left|p_{n}-q_{n}\right|}=\frac{d\left(w_{n}, q_{n}\right) \cdot d\left(p_{n}, v_{n}\right)}{d\left(w_{n}, v_{n}\right) \cdot d\left(p_{n}, q_{n}\right)} \leqslant \frac{2 \cdot 2}{d \cdot d} .
$$

Hence, $f=g$.

4. Some properties of general convergence. Theorem 2.3B describes an important property of strong convergence. We prove a similar result for general convergence

THEOREM 4.1. If $K\left(a_{n} / b_{n}\right)$ converges generally to a finite value $f$, then $\lim S_{n}\left(w_{n}\right)$ $=$ ffor every sequence $\left\{w_{n}\right\}$ such that

$$
\liminf _{n \rightarrow \infty} d\left(-h_{n}, w_{n}\right)>0 \quad\left(h_{n} \text { as given by }(3.8)\right) \text {. }
$$


Proof. Assume that for some $N \in \mathbf{N}$ and $d>0$

$$
f=\lim S_{n}\left(u_{n}\right)=\lim S_{n}\left(v_{n}\right), \quad d\left(u_{n}, v_{n}\right) \geqslant d, \quad n \geqslant N .
$$

Since $f \neq \infty$, it follows that $S_{n}\left(u_{n}\right) \neq \infty, S_{n}\left(v_{n}\right) \neq \infty$ from some $n$ on, and

$$
\left|S_{n}\left(u_{n}\right)-S_{n}\left(v_{n}\right)\right|=\frac{\left|\Delta_{n}\right|\left|v_{n}-u_{n}\right|}{\left|B_{n}+B_{n-1} v_{n}\right|\left|B_{n}+B_{n-1} u_{n}\right|} \rightarrow 0 .
$$

It suffices to prove that $\left|S_{n}\left(w_{n}\right)-S_{n}\left(u_{n}\right)\right| \rightarrow 0$. This clearly holds if

$$
\begin{aligned}
P_{n} & =\left|\frac{S_{n}\left(w_{n}\right)-S_{n}\left(u_{n}\right)}{S_{n}\left(u_{n}\right)-S_{n}\left(v_{n}\right)}\right|=\left|\frac{w_{n}-u_{n}}{u_{n}-v_{n}}\right|\left|\frac{B_{n}+B_{n-1} v_{n}}{B_{n}+B_{n-1} w_{n}}\right| \\
& =\left|\frac{w_{n}-u_{n}}{u_{n}-v_{n}}\right|\left|\frac{h_{n}+v_{n}}{h_{n}+w_{n}}\right|=\frac{d\left(w_{n}, u_{n}\right) \cdot d\left(-h_{n}, v_{n}\right)}{d\left(u_{n}, v_{n}\right) \cdot d\left(-h_{n}, w_{n}\right)} \leqslant M
\end{aligned}
$$

for all sufficiently large $n$ such that $w_{n} \neq v_{n}, u_{n}$, for some $M>0$. $\left(\left|B_{n}+B_{n-1} u_{n}\right| \neq 0\right.$ for sufficiently large $n$ since $f=\lim S_{n}\left(u_{n}\right) \neq \infty$. We can cancel $B_{n-1}$ in view of (3.7), since $v_{n}, w_{n}$ and $-h_{n}$ are distinct for sufficiently large $n$ such that $w_{n} \neq v_{n}, u_{n}$.) By (4.1) and (4.2) it follows that

$$
P_{n} \leqslant \frac{2 \cdot 2}{d \cdot c}=M<\infty \text { for sufficiently large } n,
$$

where $c=\frac{1}{2} \liminf d\left(-h_{n}, w_{n}\right)>0$.

If $f=\infty$, then

$$
\begin{aligned}
f^{(1)} & =\lim _{n \rightarrow \infty} S_{n}^{(1)}\left(v_{n+1}\right)=\lim _{n \rightarrow \infty} \frac{a_{2}}{b_{2}}+\cdots+\frac{a_{n+1}}{b_{n+1}+v_{n+1}} \\
& =-b_{1}+\frac{a_{1}}{f} \neq \infty,
\end{aligned}
$$

and we can apply Theorem 4.1 to the first tail $K\left(a_{1+n} / b_{1+n}\right)$ of $K\left(a_{n} / b_{n}\right)$. Condition (4.1) must then be replaced by

$$
\liminf d\left(-h_{n}^{(1)}, w_{n+1}\right)>0 \text { if } f=\infty,
$$

where $h_{n}^{(1)}$ belongs to $K\left(a_{1+n} / b_{1+n}\right)$. From (3.4) it follows that

$$
h_{n}^{(1)}=b_{n+1}+\frac{a_{n+1}}{b_{n}}+\frac{a_{n}}{b_{n-1}}+\cdots+\frac{a_{3}}{b_{2}}=\frac{A_{n+1}}{A_{n}}=k_{n+1},
$$

where $A_{n}$ is given by (3.3)-(3.5). But we can improve Theorem 4.1 even more. To do that we shall define some new concepts.

In [9] Waadeland introduced the concept of right and wrong tails of a continued fraction: A sequence $\left\{g^{(n)}\right\}_{n=0}^{\infty}, g^{(n)} \in \hat{\mathbf{C}}$, satisfying

$$
g^{(n-1)}=a_{n} /\left(b_{n}+g^{(n)}\right), \quad n \geqslant 1,
$$

is called the sequence of right tails of $K\left(a_{n} / b_{n}\right)$ if $K\left(a_{n} / b_{n}\right)$ converges to $g^{(0)}$; otherwise it is called a sequence of wrong tails of $K\left(a_{n} / b_{n}\right)$. It is natural to define a similar concept connected with general convergence;

Definition 4.2. A sequence $\left\{g^{(n)}\right\}_{n=0}^{\infty}, g^{(n)} \in \hat{\mathbf{C}}$, satisfying

$$
g^{(n-1)}=a_{n} /\left(b_{n}+g^{(n)}\right), \quad n \geqslant 1, a_{n} \in \mathbf{C} \backslash\{0\}, b_{n} \in \mathbf{C},
$$


is called the sequence of generally right tails (g-right tails) of $K\left(a_{n} / b_{n}\right)$, if $K\left(a_{n} / b_{n}\right)$ converges generally to $g^{(0)}$. Otherwise it is called a sequence of generally wrong tails (g-wrong tails) of $K\left(a_{n} / b_{n}\right)$.

(I am indebted to Professor W. B. Jones for suggesting the names of these new concepts.)

We see that $\left\{-h_{n}\right\}$ is a sequence of g-wrong tails of $K\left(a_{n} / b_{n}\right)$ if $K\left(a_{n} / b_{n}\right)$ does not converge generally to $-h_{0}=\infty$. Similarly, $\left\{-k_{n}\right\}$ is a sequence of g-wrong tails of $K\left(a_{n} / b_{n}\right)$ if $K\left(a_{n} / b_{n}\right)$ does not converge generally to $-k_{0}=0$.

From Theorem 4.1 and the subsequent remark on the case $f=\infty$, the next result follows:

THEOREM 4.3. Let $K\left(a_{n} / b_{n}\right)$ converge generally to $f \in \hat{\mathbf{C}}$, and let $\left\{v_{n}\right\}$ and $\left\{w_{n}\right\}$ be two sequences of modifying factors such that neither $\left\{S_{n}\left(v_{n}\right)\right\}$ nor $\left\{S_{n}\left(w_{n}\right)\right\}$ has a limit point at $f$. Then

$$
d\left(w_{n}, v_{n}\right) \rightarrow 0 \text { as } n \rightarrow \infty
$$

Proof. It suffices to prove that

$$
\begin{array}{ll}
d\left(w_{n},-h_{n}\right) \rightarrow 0 & \text { if } f \neq \infty, \\
d\left(w_{n},-k_{n}\right) \rightarrow 0 & \text { if } f=\infty .
\end{array}
$$

Assume that $f \neq \infty$. Assume further that there exists a subsequence $\left\{n_{k}\right\}_{k=1}^{\infty}$ of the natural numbers such that

$$
\liminf _{k \rightarrow \infty} d\left(w_{n_{k}},-h_{n_{k}}\right)>0 .
$$

From the proof of Theorem 4.1 it then follows that

$$
\lim _{k \rightarrow \infty} S_{n_{k}}\left(w_{n_{k}}\right)=f \text {. }
$$

This is a contradiction. Hence, no such subsequence $\left\{n_{k}\right\}$ exists, and (4.11) follows.

The proof of (4.12) is similar.

As special examples of $\left\{w_{n}\right\}$ or $\left\{v_{n}\right\}$ in Theorem 4.3 we have the sequences of g-wrong tails $\left\{g^{(n)}\right\}$, since for these it follows by (4.9) that $S_{n}\left(g^{(n)}\right)=g^{(0)}$ for all $n$. Hence, we have the following corollary to Theorem 4.3:

COROllary 4.4. Let $K\left(a_{n} / b_{n}\right)$ converge generally to $f \in \hat{\mathbf{C}}$, and let $\left\{g^{(n)}\right\}$ and $\left\{p^{(n)}\right\}$ be two sequences of $g$-wrong tails of $K\left(a_{n} / b_{n}\right)$. Then

$$
d\left(g^{(n)}, p^{(n)}\right) \rightarrow 0 \quad \text { as } n \rightarrow \infty .
$$

From this corollary it follows that the actual choice of $h_{n}$ in (4.1) or $h_{n}^{(1)}=k_{n+1}$ in (4.7) (the condition of Theorem 4.1) is not important, as long as it is a sequence of g-wrong tails. Indeed, we can state Theorem 4.1 in the following, more general form.

THEOREM 4.5. If $K\left(a_{n} / b_{n}\right)$ converges generally to $f \in \hat{\mathbf{C}}$, then $\lim S_{n}\left(w_{n}\right)=f$ for every sequence $\left\{w_{n}\right\}$ such that

$$
\liminf _{n \rightarrow \infty} d\left(g^{(n)}, w_{n}\right)>0
$$

where $\left\{g^{(n)}\right\}$ is a sequence of $g$-wrong tails of $K\left(a_{n} / b_{n}\right)$. 
With $L$ as given in Definition 2.2(ii), we clearly get the following two corollaries to Theorem 4.5 or 4.1, which connect these theorems to Theorem 2.3.

COROLlaRY 4.6. If $K\left(a_{n} / b_{n}\right)$ converges generally to a finite value $f$, then $\lim S_{n}\left(w_{n}\right)=$ ffor every sequence $\left\{w_{n}\right\}$ such that

$$
\liminf _{n \rightarrow \infty} \operatorname{dist}\left(w_{n}, L\right)>0 \text {. }
$$

Here, $\operatorname{dist}(x, L)$ is meant to be the distance on the Riemann sphere; that is,

$$
\operatorname{dist}(x, L)=\inf \{d(x, w) ; w \in L\} .
$$

COROLlaRY 4.7. If $K\left(a_{n} / b_{n}\right)$ converges generally to a finite value $f$, then $\lim S_{n}(w)$ $=f$ for every $w \in \hat{\mathbf{C}}$ such that $\operatorname{dist}(w, L)>0$.

Theorem 4.3 (or Corollary 4.4) generalizes a previous result on right and wrong tails [2]. And just as [2, Theorem 4.1] has a converse, so has Corollary 4.4.

THEOREM 4.8. Assume that $K\left(a_{n} / b_{n}\right)$ has three distinct sequences $\left\{f^{(n)}\right\},\left\{g^{(n)}\right\}$ and $\left\{p^{(n)}\right\}$ of g-right or $g$-wrong tails such that

$$
d\left(g^{(n)}, p^{(n)}\right) \rightarrow 0 \quad \text { and } \quad \liminf d\left(f^{(n)}, g^{(n)}\right)>0 .
$$

Then $K\left(a_{n} / b_{n}\right)$ converges generally to $f^{(0)}$.

Proof. Without loss of generality we assume that $f^{(0)} \neq \infty$ and $g^{(0)} \neq \infty$. (If $f^{(0)}=\infty$, we can study $K\left(a_{1+n} / b_{1+n}\right)$.) Let $\left\{w_{n}\right\}$ be a sequence of complex numbers such that

$$
d\left(w_{n},-h_{n}\right) \geqslant \varepsilon, \quad d\left(w_{n}, f^{(n)}\right) \geqslant \varepsilon \quad \text { for all } n,
$$

for an $\varepsilon>0$. Since $S_{n}\left(f^{(n)}\right)=f^{(0)} \rightarrow f^{(0)}$, it suffices to prove that $S_{n}\left(w_{n}\right) \rightarrow f^{(0)}$. That is, it suffices to prove that

$$
\begin{aligned}
P_{n} & =\left|\frac{S_{n}\left(w_{n}\right)-f^{(0)}}{f^{(0)}-g^{(0)}}\right|=\left|\frac{S_{n}\left(w_{n}\right)-S_{n}\left(f^{(n)}\right)}{S_{n}\left(f^{(n)}\right)-S_{n}\left(g^{(n)}\right)}\right| \\
& =\left|\frac{w_{n}-f^{(n)}}{f^{(n)}-g^{(n)}}\right|\left|\frac{h_{n}+g^{(n)}}{h_{n}+w_{n}}\right| \\
& =\frac{d\left(w_{n}, f^{(n)}\right) \cdot d\left(-h_{n}, g^{(n)}\right)}{d\left(f^{(n)}, g^{(n)}\right) \cdot d\left(-h_{n}, w_{n}\right)} \rightarrow 0 .
\end{aligned}
$$

(Observe that $S_{n}\left(w_{n}\right) \neq \infty$ since $w_{n} \neq-h_{n}$. Moreover, $g^{(n)} \neq-h_{n}$ since $g^{(0)} \neq \infty$. Hence, the operations in (4.19) hold.) In view of (4.17) and (4.18) and the fact that $d(\cdot, \cdot) \leqslant 2$, it suffices to prove that

$$
d\left(-h_{n}, g^{(n)}\right) \rightarrow 0
$$

If $p^{(0)}=\infty$, then $p^{(n)}=-h_{n}$ for all $n$, and (4.20) follows directly from (4.17). 
Assume that $p^{(0)} \neq \infty$. Then, for each $n \in \mathbf{N}, g^{(n)}, p^{(n)}, f^{(n)}$ and $-h_{n}$ are all distinct. We therefore get

$$
\begin{aligned}
0 & <\left|\frac{g^{(0)}-p^{(0)}}{f^{(0)}-p^{(0)}}\right|=\left|\frac{g^{(n)}-p^{(n)}}{f^{(n)}-p^{(n)}}\right|\left|\frac{h_{n}+f^{(n)}}{h_{n}+g^{(n)}}\right| \\
& =\frac{d\left(g^{(n)}, p^{(n)}\right) \cdot d\left(-h_{n}, f^{(n)}\right)}{d\left(f^{(n)}, p^{(n)}\right) \cdot d\left(-h_{n}, g^{(n)}\right)} \\
& \leqslant \frac{2}{\delta} \frac{d\left(g^{(n)}, p^{(n)}\right)}{d\left(-h_{n}, g^{(n)}\right)}
\end{aligned}
$$

for sufficiently large $n$, where

$$
\delta=\frac{1}{2} \liminf d\left(f^{(n)}, p^{(n)}\right)>0
$$

by (4.17). Since $d\left(g^{(n)}, p^{(n)}\right) \rightarrow 0,(4.20)$ follows. This proves the theorem.

\section{REFERENCES}

1. Lars V. Ahlfors, Complex analysis, 3rd ed., McGraw-Hill, New York, 1979.

2. Marcel G. de Bruin and Lisa Jacobsen, The dominance concept for linear recurrence relations with applications to continued fractions (to appear).

3. H. Hamburger, Über eine Erweiterung des Stieltjesschen Momentproblems. I, Math. Ann. 81 (1920), 235-319.

4. G. Hamel, Über einen limitärperiodischen Kettenbruch, Arch. Math. Phys. 27 (1918), 37-43.

5. Lisa Jacobsen, Modified approximants for continued fractions. Construction and applications, Det Kgl. Norske Vid. Selsk. Skr. No. 3 (1983), 1-46.

6. William B. Jones and W. J. Thron, Continued fractions. Analytic theory and applications, Encyclopedia of Mathematics and its applications, Vol. 11, Addison-Wesley, Reading, Mass., 1980.

7. Oskar Perron, Die Lehre von den Kettenbrüchen, Bd. II, Teubner, Stuttgart, 1957.

8. W. J. Thron and Haakon Waadeland, Modifications of continued fractions. A survev, Lecture Notes in Math., vol. 932, Springer-Verlag, Berlin and New York, 1982, pp. 38-66.

9. Haakon Waadeland, Tales about tails, Proc. Amer. Math. Soc. 90 (1984), 57-64.

Institute of Mathematics and Statistics, University of Trondheim, AVH, N-7000 Trondheim, NORWAY 